\title{
Tratamiento de la Ansiedad en Pacientes Prequirúrgicos
}

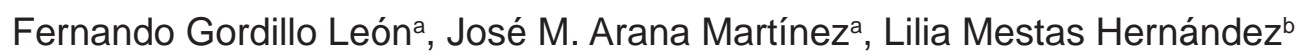

a Departamento de Psicología Básica, Psicobiología y Metodología. Universidad de Salamanca (España).

${ }^{\mathrm{b}}$ Facultad de Estudios Superiores Zaragoza. Universidad Nacional Autónoma de México (México).

Correspondencia: Fernando Gordillo León. C/ Pinzón no 4 , $2^{\circ}-\mathrm{D}, 28025$ - Madrid, España. Telf.: +34662472038 , correo electrónico: fgordilloleon@ hotmail.com.

Recibido el 30 de julio de 2011.

Aceptado para su publicación el 8 de agosto de 2011.

\section{RESUMEN}

La ansiedad en pacientes que tienen que someterse a una operación quirúrgica conlleva un estado emocional negativo y genera una activación fisiológica en la preparación del organismo para afrontar el peligro percibido en dicha operación que puede perjudicar el correcto desarrollo del procedimiento quirúrgico. Este trabajo pretende determinar qué estrategias son las adecuadas en el ámbito hospitalario para disminuir los niveles de ansiedad en los pacientes prequirúrgicos. A partir de los datos consultados se puede concluir que para evitar niveles de ansiedad perjudiciales se requiere un trato personalizado, un modelo de comunicación adecuado entre el personal sanitario y los pacientes, enseñar al enfermo el manejo de un locus de control interno y entender la infraestructura y la rutina hospitalaria a partir de las necesidades de los usuarios, sin menoscabo de los requerimientos que se determinen para el correcto funcionamiento del centro de salud.

Palabras clave. Ansiedad, Cirugía, Emociones.

\section{ABSTRACT}

\section{Treatment of anxiety in preoperative patients}

Anxiety in patients who undergo a surgical procedure entails negative emotional states and generates physiological activity that prepares the body to cope with the perceived danger: this can affect the smooth development of the surgical procedure. This study aims to determine what actions are appropriate to reduce levels of anxiety in preoperative patients. From the data consulted we conclude that personalized treatment is required to prevent harmful levels of anxiety; a good model of communication between medical staff and patients; teaching the patient the management of an internal locus of control, and understand hospital infrastructure and routine from the viewpoint of patients' needs, without compromising the proper functioning of the health center.

Key words. Anxiety, Surgery, Emotions.

\section{INTRODUCCIÓN}

La ansiedad se define como un estado en el que se experimenta un sentimiento de incomodidad que con frecuencia es inespecífico o desconocido para el individuo ${ }^{1}$; también como una reacción compleja frente a situaciones 0 estímulos actuales, potencial o subjetivamente percibidos como peligrosos, aunque sólo sea por la circunstancia de parecer inciertos ${ }^{2}$. Para el manejo de este concepto en el contexto hospitalario, el individuo debe ser visto de manera global como un organismo de gran complejidad cognitiva a través de factores como el físico, psíquico, social y espiritual ${ }^{3}$ que pudieran estar operando como mediadores o protectores de la ansiedad. Tener en cuenta estos aspectos resulta importante dado que algunos estudios reportan porcentajes de diagnóstico y niveles de ansiedad elevados en pacientes prequirúrgicos ${ }^{4,5}$.

El factor físico comprende la amenaza experimentada por el individuo en acciones de rutina hospitalaria, como la integridad cutánea, la inmovilidad, la deprivación del sueño y la agresión fisiológica. El factor psíquico es el 
más directamente relacionado con la ansiedad que experimenta el enfermo, ya que esta ansiedad surge de la pérdida de intimidad, de la necesidad de afrontar una situación desconocida, de la incertidumbre presente y futura. Los factores sociales pueden resultar atenuadores si están adecuadamente consolidados en el individuo, mediante redes familiares y de amistad con una relación de cariño y comprensión adecuada. Por otro lado, la espiritualidad parece ser beneficiosa a todo tipo de pacientes ${ }^{6}$.

La intensidad de la ansiedad frente a una intervención quirúrgica viene determinada por diversos factores ${ }^{7}$. El primero es la magnitud del agente traumático externo o real, es decir, la gravedad de la enfermedad. Otro factor importante a tener en cuenta es la duración del periodo preoperatorio: cuanto mayor sea la urgencia de la operación menor será el tiempo que tendrá el enfermo para adaptarse emocionalmente, lo que dará lugar a crisis de ansiedad y muchas manifestaciones somáticas y neurovegetativas. Los antecedentes familiares sobre intervenciones, como problemas con la anestesia o experiencias pasadas con pérdida de algún ser querido, suelen ser una de las principales fuentes de ansiedad. El último factor es la capacidad subjetiva del paciente para hacer frente a la ansiedad. Este es un factor determinante que casi siempre suelen pasar por alto los profesionales de la medicina. Intervenciones de "pequeña importancia" o "rutinarias" pueden suponer en pacientes con una débil estructura emocional un verdadero escollo, que, de no tenerse en cuenta, repercutirá en la recuperación, facilitando la aparición de complicaciones que a priori parecían de escasa probabilidad.

\section{IMPACTO EMOCIONAL DE LA INFORMACIÓN MÉDICA}

Algunos estudios evidencian que la información médica incrementa los niveles de ansiedad ${ }^{8,9}$. Sin embargo, uno de los trabajos más significativos y que contradice esta idea es el de Rodríguez, Gómez y Fernández-Crehuet ${ }^{10}$, en el que se realizó un estudio sobre 220 pacientes operados por diferentes patologías (urología, cirugía de mama y endocrina, y cirugía digestiva). A todos se les aplicó una prueba para la evaluación de estado mental (MMSE: Mini Mental State Examination) ${ }^{11}$, otra para medir la ansiedad (STAI: State-Trait Anxiety
Inventory) ${ }^{12}$, un cuestionario de personalidad (EPI: Eysenck Personality Inventory $)^{13}$ y un cuestionario general de evaluación de actitud del paciente hacia el proceso de consentimiento informado (cuestionario general $\mathrm{Cl})^{10}$. Los resultados muestran que el $82,3 \%$ de los pacientes informaron no haber tenido sensación subjetiva de ansiedad al recibir la información médica, frente al 16,8\% que afirmaban haberse puesto nerviosos, y el 0,9\% que decían haberlo estado mucho. El 87,7\% dijo preferir saber en todo momento lo que les pasaba y lo que se les iba a hacer. Uno de los datos más significativos es el hecho de que el $91,3 \%$ de los pacientes que puntuaron alto en el STAI-Estado no recibieron información alguna por parte del médico sobre la intervención a la que iban a ser sometidos. Por otra parte el 93,3\% de los sujetos a los que se les explicó en qué consistiría la intervención puntuaron normal o bajo en la STAI-Rasgo. Por último, se contrastó la variable EPI con la variable "ansiedad subjetiva", encontrándose que el rasgo de personalidad que correlacionaba significativamente con el estado de ansiedad subjetiva era el neuroticismo. Sujetos con puntuaciones altas en neuroticismo presentan dificultades para recuperarse después de una situación emocional estresante.

Otro de los efectos de la ansiedad en los pacientes quirúrgicos tiene que ver con la comunicación entre paciente y médico. Ya no se trata de si la información que pueda suministrar el médico tendrá efectos beneficiosos o no; la cuestión es que la ansiedad impide que el paciente pueda comprender adecuada, objetiva y razonadamente las instrucciones y descripciones sobre su enfermedad. Podríamos decir que la ansiedad provoca una falta de comunicación. Sucede como si paciente y médico, de pronto, hablaran idiomas distintos. Este ha sido el tema de un trabajo de investigación realizado por Atchison et al. ${ }^{14}$, donde se comprobó que en pacientes con problemas de comunicación (bien por su nivel cultural, o bien por ser inmigrantes) su adecuación al tratamiento postoperatorio venía determinada por el nivel de comprensión de las instrucciones dadas por el médico. Mediante la utilización de traductores e instrucciones detalladas se consiguió mayor adherencia a los tratamientos.

Otro ejemplo de los beneficios de la comunicación viene dado en un estudio realizado por Arslan et al. ${ }^{15}$, en el que a sujetos a los que se les iba a practicar por primera vez una rinoplastia se les preguntó por el 
momento del proceso quirúrgico en el que sintieron mayor ansiedad. Se informa que los efectos de la ansiedad podían ser atenuados en la fase preoperatoria mediante una información detallada de la operación a la que iban a ser sometidos y la administración de algún tranquilizante, en los casos en los que fuera necesario. De igual manera, un procedimiento de visita prequirúrgica estructurada de enfermería en el que el paciente recibía información adicional sobre el proceso quirúrgico produjo una disminución de los niveles de ansiedad situacional ${ }^{16}$.

El estado de ansiedad preoperatoria debería ser tenido en cuenta más allá de la información verbal que el propio paciente suministre al médico. Es habitual que los anestesistas visiten a los pacientes con la intención de conocer su estado anímico, pero quizá no sean del todo conscientes de las repercusiones que puede tener el no advertir un estado de ansiedad desmedido. Frases como "Quédese tranquilo, es una operación sin importancia, no pasará nada", pueden dejar al paciente con la sensación de que no ha sido escuchado. Las manifestaciones no verbales son muy interesantes a la hora de evaluar el nivel de ansiedad ${ }^{17}$ : temblor, sudoración, expresión de la cara, etc. También deben estar atentos a los cambios fisiológicos que acompañan a estos estados de ansiedad, como cambios en la tensión arterial o en la frecuencia cardiaca, ya que pueden afectar a la respuesta de las drogas anestésicas. En este sentido, los síntomas que alertan de un nivel de ansiedad moderado son: insomnio, pesadillas, cefaleas tensionales, náuseas, dispepsia, sensación de disnea u opresión precortical atípica; muy significativos son los retrasos excusados por el paciente para el día de la operación, casi siempre relacionados con problemas de origen somático, como reacciones alérgicas, resfriados, etc.

De lo expuesto en este apartado se puede concluir que la evaluación de la ansiedad es un paso previo a su control y, como hasta ahora hemos visto, este control se hace imprescindible si queremos mejorar las condiciones pre y post operatorias, además de los beneficios que aportaría al desarrollo de la propia operación (variables fisiológicas, respuesta a los antibióticos, sedantes, etc.). En ese sentido, hay que tener en cuenta tanto aspectos internos (locus de control) como externos (arquitectura, rutina y decoración hospitalaria) del sujeto.

\section{LOCUS DE CONTROL}

Algunas personas explican un acontecimiento como contingente a su propia conducta, mientras otras lo hacen como fuerzas ajenas a ella. Los primeros tiene un locus de control interno, los segundos externo. Rotter concibió el concepto como una variable unidimensional, con dos valores: internalidad y externalidad ${ }^{18}$.

El control percibido modula la reactividad psicobiológica en los estados emocionales ${ }^{19}$, y por consiguiente el control de la ansiedad vendría determinado por el control percibido de la situación que los pacientes quirúrgicos tienen. En definitiva, aquellas personas que crean que su salud depende en primera instancia de ellas mismas (locus de control interno) tendrán mejor salud que aquellas que crean que las causas de la enfermedad son ajenas a la persona que las sufre (locus de control externo ${ }^{20}$. Sin embargo, Wallston admitió que su hipótesis contenía algunas imprecisiones, por lo que decidió cambiar el término de locus control por el de "Competencia Personal Percibida", es decir, que se podrá conseguir una motivación adecuada para llevar a cabo conductas que mantengan la salud cuando esta percepción de lo competente que es uno sea lo suficientemente alta. Tener una competencia personal alta consiste en valorar la salud como un bien importante y creer que se es capaz de realizar conductas saludables. En realidad lo que hace Wallston al modificar el término es acercarse al concepto de autoeficacia de Bandura ${ }^{21}$.

Este planteamiento abre muchas posibilidades al tratamiento de la ansiedad en términos de control percibido. En este sentido Eifert, Coburn y Seville22 piensan que los procesos de extinción, habituación, inhibición recíproca o contracondicionamiento no dan cuenta de todos los aspectos de los métodos clínicos de reducción de la ansiedad utilizados hasta la fecha. Los autores sostienen que los cambios en la percepción de control son los responsables en la mejora de los trastornos de ansiedad y que para superar este tipo de trastornos son cruciales los siguientes factores: a) respuestas fisiológicas en situaciones de ansiedad; b) estímulos o situaciones que provocan la ansiedad; c) la propia conducta de exposición a dichos estímulos o situaciones. En el trabajo realizado por Sorlie y Sexton ${ }^{23}$ se constató que las intervenciones enfocadas al cambio de percepción del locus de control hacia la internalidad 
son beneficiosas. Se encontraron correlaciones positivas entre los niveles de alta internalidad obtenidos en el test Multidimensional Health Locus Control (MHLC), bajo nivel de ansiedad y mejor adherencia al tratamiento.

\section{ARQUITECTURA, RUTINA Y DECORACIÓN HOSPITALARIA}

El hospital no debería convertirse en un establecimiento estancado apartado de la vida social. Al contrario, debería ser lugar en el que curar y rehabilitar al enfermo tanto física como mentalmente, preparándole para los posibles cambios que pudieran derivarse de la enfermedad con la que ingresó. Esto se evidencia mucho más en pacientes pediátricos, que mantienen un umbral de ansiedad mucho más bajo que el paciente adulto, incrementado porque no llegan a comprender muchas veces la necesidad de la intervención quirúrgica. En un trabajo realizado por el Departamento de Personalidad de la Universidad de Murcia $^{24}$ se analizó el nivel de ansiedad experimentado con la implantación del procedimiento de cirugía sin ingreso o ambulatoria (UCSI), que consiste en dar de alta al niño el mismo día de la operación, sin necesidad de dejarle hospitalizado. Los momentos elegidos para medir el nivel de ansiedad fueron cinco: 1. Unos días antes de la operación. 2. La mañana de la operación. 3. Bajando al quirófano. 4. La tarde después de la operación. 5. Varios días después de la operación. Los resultados no encontraron diferencias significativas entre el grupo de control y el experimental. Estos resultados evidencian que no resulta efectivo, salvo económicamente, reducir la estancia de los pacientes en el centro. Lo más conveniente sería adecuar la infraestructura hospitalaria al usuario.

La arquitectura y la decoración hospitalaria influyen en el proceso de recuperación posquirúrgico y reducen el nivel de ansiedad prequirúrgico. Un estudio realizado por Ulrich ${ }^{25}$ demostró que aquellos pacientes que se encontraban con vistas a un paisaje necesitaron menos analgésicos y menos días para ser dados de alta. Los colores son estímulos que pueden producir excitación o sosiego. Los hospitales deberían valerse de estos datos para validar un espectro de ambientes acorde al tipo de paciente, así como tener en cuenta las entradas lumínicas, por cuanto la luz es necesaria para mantener los relojes biológicos, y estos son un centro fundamental de bienestar que controlan importantes sistemas hormonales $y$ nerviosos. Además, una mayor flexibilidad en los horarios, mayor información en forma de visitas por parte del personal médico, así como actividades que se salgan de la norma, han demostrado ser beneficiosos para reducir la ansiedad prequirúrgica.

\section{CONCLUSIÓN}

Los estados de ansiedad elevados en los periodos previos a la operación quirúrgica se ven reflejados durante la operación, ya que el cuerpo reacciona de diferente forma ante la anestesia en estado de gran ansiedad, y en los periodos postoperatorios, retardando la recuperación y retrasando el abandono del centro hospitalario, lo que podría producir saturación en el centro hospitalario y un mayor costo económico. Comprender las variables que afectan a este incremento de la ansiedad antes de la operación resulta de vital importancia para su tratamiento. A este respecto realizamos las siguientes recomendaciones:

Tratar individualizadamente los trastornos de ansiedad en el ámbito hospitalario. Si bien la aplicación de un protocolo que ayude al profesional es efectivo, nunca debe ir separado de una entrevista personal que le permita observar las reacciones del paciente, evitando que se pasen por alto estados de ansiedad no reflejados en los cuestionarios estándar. En este sentido es recomendable conocer el tipo de personalidad del paciente mediante la aplicación de tests que permitan la mejora en la efectividad de los tratamientos psicológicos orientados a reducir la ansiedad preoperatoria.

- Enseñar a los profesionales sanitarios el modo de comunicarse con los enfermos de la manera más precisa, efectiva e inocua, evitando el estrés que supondría una mala información, o la ausencia de la misma ${ }^{26}$.

- Hacer partícipe al enfermo de su recuperación. Tener en cuenta la forma habitual que tiene el sujeto de explicar los acontecimiento vitales, en otras palabras: conocer primero donde se sitúa el locus del paciente, para optimizar la terapia psicológica, si fuera necesaria, para reducir los niveles de ansiedad. 
- Adecuar el espacio hospitalario a las necesidades del enfermo, así como fomentar el conocimiento de la rutina hospitalaria, que ha demostrado ser útil en la reducción de la ansiedad $^{27}$.

- Modelo ecológico de información y formación del paciente. Atender a la diferencia entre informar y educar a los pacientes para permitir un afrontamiento del proceso quirúrgico que minimice los niveles de ansiedad. La información tiene un efecto a corto plazo que, como hemos visto, no siempre resulta efectivo, sin embargo, la formación no sólo de los pacientes, también de los familiares y amigos, permitirá el tratamiento de la ansiedad del paciente durante su estancia hospitalaria y su prevención fuera del hospital, fomentando hábitos saludables y estrategias de afrontamiento que repercutirán en todas las variables intrahospitalarias analizadas en este trabajo.

Es conveniente tener en cuenta que la aplicación de medidas que a nivel teórico parecen congruentes con los objetivos buscados no siempre son fáciles de llevar a término. Este trabajo y sus conclusiones son un claro ejemplo de esta premisa. Las largas jornadas laborales del personal sanitario, el estrés consecuente al tipo de trabajo realizado y el alto grado de emocionalidad presente en toda interacción con los enfermos y sus familiares hace inviable la posibilidad de que sean los médicos y/o el personal de enfermería los encargados de diagnosticar un posible trastorno psiquiátrico en pacientes que acuden por otro tipo de enfermedades. Por esta razón, resulta de vital importancia el papel del psicólogo en los centros hospitalarios como parte del equipo sanitario.

\section{BIBLIOGRAFÍA}

1. Alfaro R. Aplicación del proceso de enfermería. Madrid: Mosby; 1996. p. 232.

2. Barrilero JA, Casero JA, Cebrián F, Córdoba CA, García F, Gregorio E, et al. Ansiedad y cirugía. Repercusiones en el paciente sometido a cirugía programada. Rev Enfermería (Albacete). 1998; 208.

3. Marín I, Martínez P, Ponsich J, Pubill M. Ansiedad en pacientes intervenidos de rodilla (Prótesis total rodilla). Enferm Global. 2004; 4:1-11.

4. Simoes Flório MC, Galván CM. Cirugía Ambulatorial: Identificáo dos diagnósticos de enfermagem no período erioperatório. Rev Latino-Am Enfermagem. 2003; $11: 630-7$.
5. Valenzuela-Millán J, Barrera-Serrano JR, Ornelas-Aguirre JM. Ansiedad preoperatoria en procedimientos Anestésicos. Cir Cir. 2010; 78:151-6.

6. Siegel K, Schrimshaw EW. The perceived benefits of religious and spiritual coping among older adults living with HIVIAIDS. Journal of scientific study of religions. 2002; 41:91-102.

7. Giacomantone E, Mejía A. Estrés quirúrgico y ansiedad. Criterios para su manejo terapéutico. Gador; 1998.

8. Alfidi R. Informed consent: a study of patient reaction. JAMA. 1971; 216:1325-9.

9. Spring D, Akin J, Margulis A. Informed consent for introvenous contrast-enhanced radiography: a national survey of practice and opinion. Radiology. 1984; 152:60913.

10. Rodríguez JC, Gómez E, Fernández-Crehuet J. El impacto emocional de la información médica en pacientes quirúrgicos. Cuadernos de Bioética. 2001; 12:367-78.

11. Folstein MF, Folstein SE, McHugh PR. Mini Mental State: A practical method for grading the cognitive state of patients for the clinician. Journal of Psychiatric Research. 1975; 12:189-98.

12. Spielberger CD, Goursch RL, Lushene RE. Cuestionario de Ansiedad Estado - Rasgo, STAI. Madrid: TEA Ediciones; 1982.

13. Eysenck S, Eysenck $\mathrm{H}$. Acquiescence response set in personality cuestionaires. Life Sciences. 1963; 2:144-7.

14. Atchison KA, Black EE, Leathers R, Belin TR, Abrego $\mathrm{M}$, Gironda $\mathrm{M}$, et al. A qualitative report of patient problems and postoperative instructions. J Oral Maxillofac Surg. 2005; 63:449-56.

15. Arslan E, Demirkan F, Unal S, Aksoy A, Polat G, Kanik A. The relationship between patient stress and the blood levels of acute phase proteins in rhinoplasty: the report of a prospective study. J Psychosom Res. 2005; 58:35-41.

16. Chirveches-Pérez E, Arnau-Bartés A, Soley-Bach M, Rosell-Cinca F, Clotet-Argemi G, Roura-Poch P, et al. Efecto de una visita prequirúrgica de enfermería perioperatoria sobre la ansiedad y el dolor. Enfermería Clínica. 2006; 16:3-10.

17. Pritchard M. Measuring anxiety in surgical patients using a visual analogue scale. Nurs Stand. 2010; 25:40-4.

18. Rotter JB, Seeman MR, Leverant S. Internal versus external control of reinforcements: A major variable inbehavior theory. En: Washburn WF, Editors. Decisions, values and groups. Vol. 2. New York: Pergamon Press; 1962. p. 473-516.

19. Fernández J, Edo S. ¿Cómo influye el control percibido en el impacto que tienen las emociones sobre la salud? Anales de Psicología. 1994; 10:127-33.

20. Wallston KA. Hocus-Pocus, the focus isn't strictty on locus: Rotter's social learning theory modified for health. Cognitive Theory and Research. 1992; 16:183-99.

21. Bandura A. The explanatory and predictive scope of selfefficacy theory. Journal of Clinical and Social Psychology. 1986; 4:359-73.

22. Eifert GH, Coburn KE, Seville JL. Putting the client in control: The perception of control in the behavioral treatment of anxiety. Anxiety, Stress, and Coping. 1992; 3:165-73.

23. Sorlie T, Sexton, HC. Predictors of change in health locus of control following surgical treatment. Personality and Individual Differences. 2004; 36:991-1004. 
24. Ortigosa JM, Méndez FX. Influencia del tipo de hospitalización en la ansiedad infantil ante la intervención quirúrgica. IV Congreso internacional sobre conductisvo y ciencias de la conducta 1998, 18-21 de Noviembre, Sevilla, España. Disponible en: http://www.um.es/gaihinfa/ resumenes/rl/rl.htm.

25. Ulrich RS. View through a window may influence recovery from surgery. Science. 1984; 224:420-1.
26. Torrico E, Santín C, Andrés M, Menéndez S, López MJ. El modelo ecológico de Bronfrenbrenner como marco teórico de la Psicooncología. Anales de Psicología. 2002; 18:4559.

27. Moix J. Emoción y cirugía. Anales de Psicología. 1998; 10:167-75. 